\title{
Um Modelo Analítico para Avaliação de Tensões em Tanques Fechados Delgados submetidos à Pressão Interna não Uniforme
}

\author{
Santos, P.R.A. ${ }^{1}$, Loeffler, C.F. ${ }^{{ }^{*}}$ \\ 1 Departamento de Engenharia Mecânica, Universidade Federal do Espírito Santo, Vitória, ES, Brasil.
}

*e-mail: carlosloeffler@bol.com.br

\section{Resumo}

\begin{abstract}
Neste trabalho elaborou-se um modelo estático teórico simplificado, baseado na teoria da membrana, aplicável a problemas de vasos pressão de paredes finas, para caracterização das tensões circunferenciais, cisalhantes e longitudinais geradas por pressão interna não uniforme, devido à contenção de líquidos em seu interior. Com a geração das equações de governo e identificação da distribuição das tensões, almeja-se posteriormente implementar uma análise vibracional, típica do problema de transporte de líquidos em tanques.
\end{abstract}

\begin{abstract}
This study presents a simplified theoretical static model based on the theory of membranes, applicable to problems of thin-walled pressure vessels, to characterize the circumferential and longitudinal stresses generated by non uniform internal pressure due to fluid retention inside. From the governing equations deduced and the identification of stress field, the future purpose is to implement a vibrational analysis, extending the model for tanks used to liquid transport.
\end{abstract}

Keywords: thin-walled pressure vessels, analytical models, membrane theory.

\section{Introdução}

Os problemas estruturais originados pela movimentação de líquidos em tanques durante o transporte são bastante importantes. Há extenso inventário de falhas por fadiga devido a continua vibração induzida a carga pelos veículos em razão de curvas, obstáculos e irregularidades na pista de tráfego, seja por meio rodoviário ou ferroviário. Não obstante 0 interesse em se identificar estatisticamente perfis de carregamento gerados no percurso ao longo das vias de transporte e aplicar métodos numéricos sofisticados para melhor avaliação do problema, é notória a deficiência de modelos matemáticos simples, que empregam teorias acessíveis da mecânica dos materiais, para estimação das tensões originadas de excitações de baixa frequência e identificação de deslocamentos obedientes aos modos mais baixos de vibração. Um modelo matemático dessa natureza é aqui deduzido, em que as condições de confinamento do líquido são aproximadas de acordo as características de um vaso fechado.

Vale ressaltar que o modelo doravante apresentado segue as hipóteses básicas da teoria de invólucros de paredes finas, que tenham a forma de uma superfície de revolução e sejam submetidos à pressão interna contínua, não sendo, porém, necessariamente uniforme [1]. A flexão das paredes é considerada desprezível, não deve haver descontinuidades geométricas e a consideração de forças ou momentos concentrados sofre restrições. A distribuição de esforços também é não válida na proximidade das condições de apoio, que são idealizadas de modo a se poder resolver analiticamente o modelo proposto.

\section{Formulação Básica}

Considere uma membrana, a qual está sujeita a carregamento assimétrico. Neste caso as tensões principais não atuam nas direções meridionais e 
tangenciais, conforme indica a Fig. 1. Três equações de equilíbrio são necessárias para descrever o comportamento do sistema, que possui duas tensões normais Sx e Sy e tensões de cisalhamento recíprocas Ss no plano local da membrana.

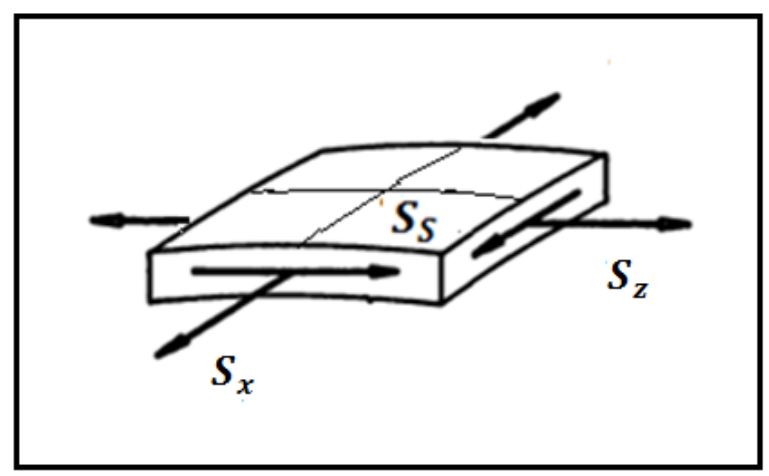

Figura 1 - Descrição do estado de tensões para carregamento assimétrico.

As equações são as seguintes [2]:

$$
\begin{gathered}
\frac{S_{x}}{R_{x}}+\frac{S_{z}}{R_{z}}=\frac{P_{i}}{t} \\
\left(\frac{\partial S_{x}}{\partial x}+\frac{\partial S_{S}}{\partial z}\right)=0 \\
\left(\frac{\partial S_{z}}{\partial z}+\frac{\partial S_{S}}{\partial x}\right)=0
\end{gathered}
$$

As direções $x$ e $z$ são quaisquer direções em que se conheçam as tensões normais atuantes e a tensão cisalhante associada. Neste modelo é necessário que a estrutura possua paredes finas, mas não se trata de análise exclusiva das tensões normais de membrana, uma vez que o efeito do cisalhamento na estrutura é contabilizado [3,4]. Na realidade, formula-se aqui um modelo mais acessível pertinente à Teoria de Cascas, com simplificações adequadas ao problema abordado.

\section{Determinação da Tensão Circunferencial}

Admite-se um vaso de pressão cilíndrico completamente cheio de líquido e sob flexão, caso típico de carregamento assimétrico, uma vez que a pressão interna varia com a altura de líquido. A figura 2 mostra o eixo longitudinal $z$, o eixo transversal y e o eixo circunferencial $x$. Este é definido na seção transversal perpendicular ao eixo do cilindro. O raio de curvatura longitudinal é infinito e o raio circunferencial é o próprio raio do vaso de pressão. Assim sendo, a equação (1) se simplifica, ou seja:

$$
S_{x}=\frac{P_{i} r}{t}
$$

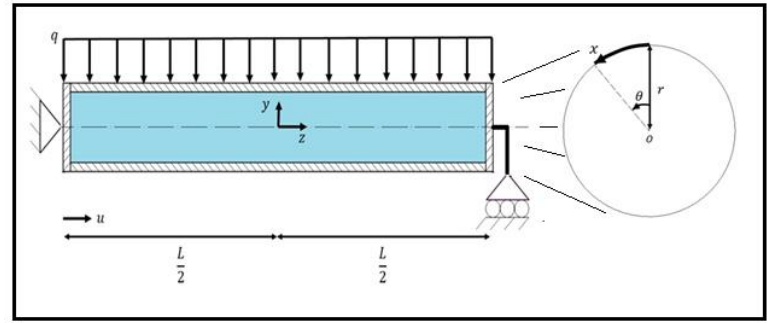

Figura 2 - Vaso de pressão cilíndrico fechado contendo líquido.

Neste caso, a pressão é uma função da coordenada y:

$$
P_{i}(y)=\rho g(r-y)+P_{\text {extra }}
$$

$\mathrm{P}_{\mathrm{i}}$ (y) é a pressão interna, que é a mesma ao longo de toda direção longitudinal; $\rho$ é a massa específica do fluido, g é a aceleração da gravidade $r$ é o raio interno

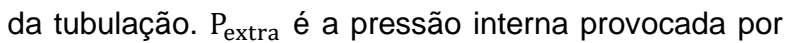
fatores não relacionados ao peso do fluido, tal como ação de bombas. Assim, tem-se:

$$
S_{x}=\frac{\left[\rho g(r-y)+P_{\text {extra }}\right] r}{t}
$$

\section{Determinação da Tensão Cisalhante}

Substituindo a Eq. (6) na Eq. (2) tem-se:

$$
\frac{\partial}{r \partial \theta} \frac{\left[\rho g(r-y)+P_{\text {extra }}\right] r}{t}+\frac{\partial S_{S}}{\partial z}=0
$$

É estratégico escrever a variável $x$ em função do ângulo $\Theta$ e posteriormente representá-lo em função da altura y, conforme o algebrismo mostrado a seguir:

$$
y=r \cos \theta \rightarrow d y=-r \operatorname{sen} \theta d \theta=-\left(r^{2}-y^{2}\right)^{\frac{1}{2}} d \theta
$$

Então, substituindo a Eq. 8 na Eq. (7), chega-se a:

$$
S_{S}=\left(-\frac{\rho g r \operatorname{sen} \theta}{t}\right) z+f_{1}(\theta)
$$

Para determinar $f_{1}(\theta)$ faz-se o estudo do esforço cortante na estrutura, pois que é o responsável pelo cisalhamento. Quando o esforço cortante é nulo (em $\mathrm{z}=0$ ) não deve existir tensão de cisalhamento; logo, a função $f_{1}(\theta)$ é nula, de tal modo que a tensão cisalhante ao longo do vaso de pressão é dada por:

$$
S_{S}=\left(-\frac{\rho g r \operatorname{sen} \theta}{t}\right) z=\left[-\frac{\rho g\left(r^{2}-y^{2}\right)^{\frac{1}{2}}}{t}\right] z
$$

\section{Determinação da Tensão Longitudinal}


Substituindo a equação (10) na equação de equilíbrio (3), e integrando, tem-se:

$$
S_{Z}=\frac{\rho g \cos \theta}{2 t} z^{2}+f_{2}(\theta)=\frac{\rho g y}{2 t r} z^{2}+f_{2}(y)
$$

Considerações de minimização de energia apontam que a função $\mathrm{f}_{2}(\mathrm{y})$ deve ser aproximada por funções mais simples [2]. Assume-se então a seguinte forma:

$$
S_{z}=\frac{\rho g y}{2 t r} z^{2}+k_{1} y+k_{2}
$$

As constantes k1 e k2 são obtidas a partir de considerações de equilíbrio envolvendo a flexão e o esforço axial reinantes no vaso. A tensão longitudinal, atuando em cada elemento de área em y, promove uma força longitudinal que por sua vez promove um momento em relação à linha neutra. O somatório de momentos provocados por cada elemento de área é o momento fletor atuante na seção. Fazendo uma parametrização do diferencial de área na forma:

$$
d A=r t d \theta=-\frac{r t}{\left(r^{2}-y^{2}\right)^{\frac{1}{2}}} d y
$$

O momento fletor pode ser calculado por:

$$
\begin{array}{r}
M_{F}=-\left(\frac{\rho g}{2 \operatorname{tr}} z^{2}+k_{1}\right) \operatorname{tr} \int_{-r}^{+r} \frac{y^{2}}{\left(r^{2}-y^{2}\right)^{\frac{1}{2}}} d y \\
-k_{2} r t \int_{-r}^{+r} \frac{y}{\left(r^{2}-y^{2}\right)^{\frac{1}{2}}} d y
\end{array}
$$

As integrais podem ser resolvidas por meio da técnica de substituição trigonométrica. Daí resulta que:

$$
M_{F}=-\left(\frac{\rho g}{2 \operatorname{tr}} z^{2}+k_{1}\right) \frac{\operatorname{tr}^{3} \pi}{2}
$$

A constante $\mathrm{k}_{1}$ é obtida pelo estudo do momento fletor, que para $\mathrm{z}=0$ vale q. $\mathrm{L}^{2} / 8$. Com isto:

$$
S_{Z}=\frac{\rho g y}{2 t r} z^{2}-\frac{q L^{2}}{4 \pi t r^{3}} y+k_{2}
$$

A constante $k_{2}$ é obtida ao realizar o estudo do somatório de forças na direção longitudinal. A pressão exercida na tampa promove uma força longitudinal que traciona as paredes do vaso de pressão fazendo surgir tensões longitudinais ao longo da seção transversal:

$$
\int \mathrm{S}_{\mathrm{Z}} \mathrm{dA} \mathrm{A}_{\text {seção }}=\int \mathrm{P}_{\mathrm{i}} \mathrm{dA} \text { tampa }
$$

$$
\begin{gathered}
\int \mathrm{S}_{\mathrm{Z}} \mathrm{dA}_{\text {seção }}=-\left(\frac{\rho \mathrm{g}}{2 \operatorname{tr}} \mathrm{z}^{2}-\frac{\mathrm{qL}}{4 \pi \mathrm{tr}^{3}}\right) \operatorname{tr} \int_{-\mathrm{r}}^{\mathrm{r}} \frac{\mathrm{y}}{\left(\mathrm{r}^{2}-\mathrm{y}^{2}\right)^{\frac{1}{2}}} \mathrm{dy} \\
-\mathrm{k}_{2} \mathrm{rt} \int_{-\mathrm{r}}^{\mathrm{r}} \frac{\mathrm{dy}}{\left(\mathrm{r}^{2}-\mathrm{y}^{2}\right)^{\frac{1}{2}}}
\end{gathered}
$$

A primeira integral é nula e a segunda é igual $\pi$. Assim, a força proveniente da tensão longitudinal na estrutura é dada de acordo por:

$$
\int \mathrm{S}_{\mathrm{Z}} \mathrm{dA}_{\text {seção }}=-\mathrm{k}_{2} \mathrm{rt \pi}
$$

Equacionando o diferencial de área da tampa, ou seja:

$$
\mathrm{d} \mathrm{A}_{\text {tampa }}=2 \mathrm{rsen} \theta \mathrm{dy}=2\left(\mathrm{r}^{2}-\mathrm{y}^{2}\right)^{\frac{1}{2}} \mathrm{dy}
$$

Com isto:

$$
\begin{aligned}
\int \mathrm{P}_{\mathrm{i}} \mathrm{dA} A_{\text {tampa }}=2( & \left.\rho g r+\mathrm{P}_{\text {extra }}\right) \int_{-r}^{\mathrm{r}}\left(\mathrm{r}^{2}-\mathrm{y}^{2}\right)^{\frac{1}{2}} \mathrm{dy} \\
- & 2 \rho g \int_{-\mathrm{r}}^{\mathrm{r}} \mathrm{y}\left(\mathrm{r}^{2}-\mathrm{y}^{2}\right)^{\frac{1}{2}} \mathrm{dy}
\end{aligned}
$$

Resolvendo:

$$
\mathrm{k}_{2}=-\frac{\mathrm{r}}{\mathrm{t}}\left(\rho \mathrm{gr}+\mathrm{P}_{\text {extra }}\right)
$$

Então, a tensão longitudinal que atua na parede do cilindro é dada por:

$$
\mathrm{S}_{\mathrm{Z}}=\frac{\rho g \mathrm{y}}{2 \mathrm{tr}} \mathrm{z}^{2}-\frac{\mathrm{qL}^{2}}{4 \pi \mathrm{tr}^{3}} \mathrm{y}-\frac{\mathrm{r}}{\mathrm{t}}\left(\rho g r+\mathrm{P}_{\text {extra }}\right)
$$

Perceba a flexão foi contabilizada, uma vez que o efeito do momento fletor e do esforço cisalhante identificaram o valor das constantes de integração.

\section{Exemplo de Aplicação}

Considere os seguintes valores, tomados de um aço inoxidável AISI 316L, com série de 30" e Schedule 20:

\begin{tabular}{|c|c|}
\hline massa específica do fluido $\left(\mathrm{kg} / \mathrm{m}^{3}\right)$ & 999 \\
\hline gravidade local $\left(\mathrm{m} / \mathrm{s}^{2}\right)$ & 9,81 \\
\hline raio interno $(\mathrm{m})$ & 0,3683 \\
\hline pressão extra $(\mathrm{Pa})$ & 0 \\
\hline espessura $(\mathrm{m})$ & 0,0127 \\
\hline massa específica da tubulação $\left(\mathrm{kg} / \mathrm{m}^{3}\right)$ & 7500 \\
\hline carregamento distribuído $(\mathrm{N} / \mathrm{m})$ & 6338,5608 \\
\hline comprimento entre apoios $(\mathrm{m})$ & 2 \\
\hline Tensão de escoamento $(\mathrm{MPa})$ & 240 \\
\hline Fator de segurança & 1 \\
\hline Tabela 1-Parâmetros para o exemplo.
\end{tabular}

Desta maneira:
Primeiramente, mostra-se o comportamento da tensão longitudinal na figura 3: 


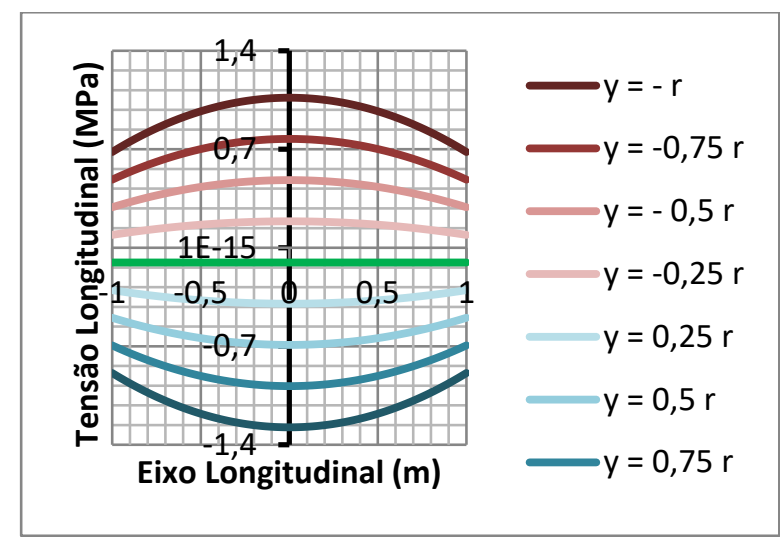

Figura 3 - Tensão de longitudinal atuante no exemplo.

Os maiores valores de tensão longitudinal se encontram no meio da tubulação, pois o momento fletor é máximo nesta localidade. Para o centróide da seção transversal a tensão longitudinal não é nula devido à assimetria do carregamento. Ao serem observadas as extremidades superior e inferior da tubulação, vê-se que o momento fletor atua tracionando as fibras inferiores e comprimindo as superiores. Nos apoios, diferentemente do caso de vigas, a tensão longitudinal não é nula. Isto ocorre pelo fato dela ser causada não somente pelo momento fletor, mas também pela pressão interna do fluido, que tensiona as tampas.

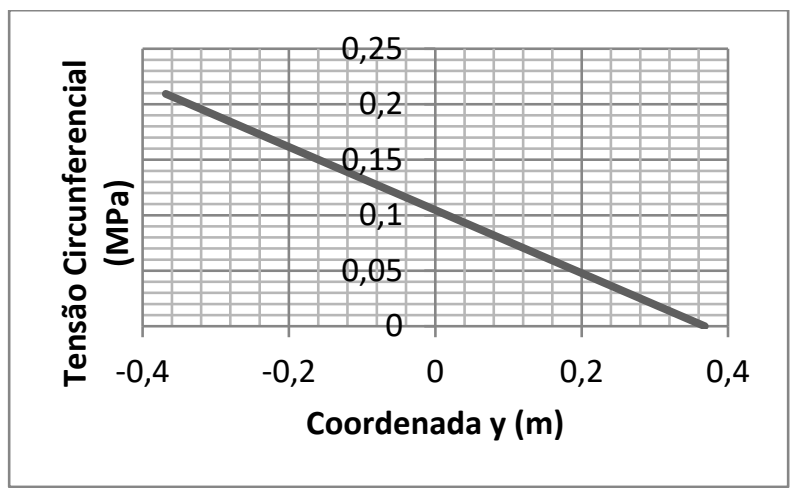

Figura 4 - Tensão circunferencial atuante no exemplo.

O comportamento da tensão circunferencial é dado na figura 4. A tensão circunferencial se deve ao efeito da pressão interna da tubulação. Isto justifica o fato da tensão ser máxima no fundo e mínima no topo. Neste caso em particular, não foi considerado qualquer pressão extra na tubulação, sendo que a pressão interna é causada exclusivamente pelo efeito do peso do fluido. Com isto, a tensão circunferencial no topo da tubulação é nula. Mas caso uma parcela extra de pressão fosse considerada, não mais esta tensão seria nula. É importante ressaltar que a tensão circunferencial não é uma função da coordenada longitudinal. Ou seja, para um mesmo valor de y, a tensão é a mesma ao longo de toda tubulação.

O comportamento da tensão de cisalhamento pode ser observado na figura 5. Esta é nula no centro da tubulação $(\mathrm{z}=0)$, pelo fato da tensão de cisalhamento ser provocada pelo esforço cortante, que é nulo nesta seção. As máximas tensões de cisalhamento ocorrem na linha neutra $(y=0)$ e as mínimas tensões ocorrem nas extremidades superior e inferior da tubulação.

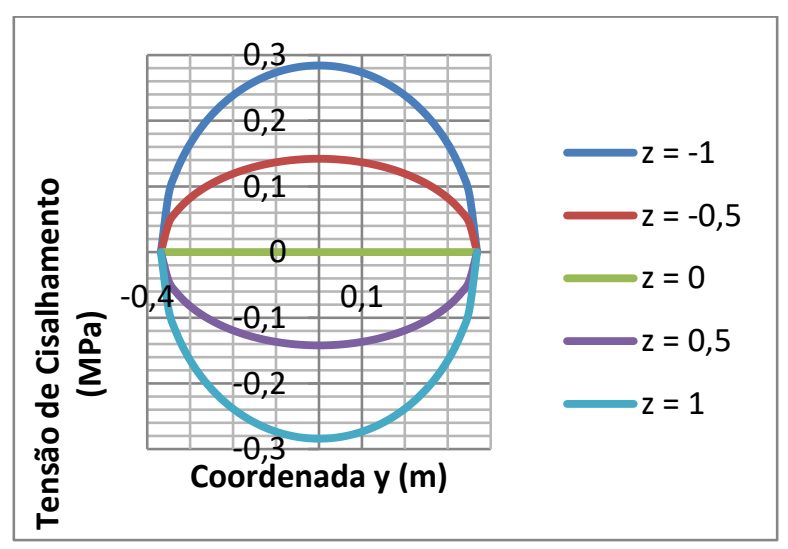

Figura 5 - Tensão cisalhante no exemplo.

\section{Conclusões}

Com a Teoria de Vigas foi possível chegar a uma solução estrutural de um problema de vaso de pressão, em que o estado de tensão produzido pelo fluido em seu interior pode ser contabilizado como função dos esforços simples atuantes no vão. O modelo aqui desenvolvido não é tão abrangente quanto os codificados nos programas computacionais, mas oferece estimadores para melhor avaliação da resposta em casos semelhantes. Ressalta-se que é viável estendê-lo a uma análise vibracional, gerando subsídios importantes para o projeto dinâmico.

$O$ efeito de assimetria do fluido em termos quantitativos foi pouco relevante numa comparação com modelos que consideram uma pressão média. Também as tensões flexionais gerada pela sustentação em vão do vaso tendem a ser pequenas em comparação com as pressões usuais consideradas na indústria de petróleo. Exceções ocorrem quando os fluidos se liquefazem à elevada pressão ou o tamanho dos vãos entre apoios e o peso próprio do vaso excede os padrões usuais.

\section{Referências}

[1] FÉODOSIEV, V. Resistência dos Materiais. Porto: Lopes da Silva Editora, Porto: 1977.

[2] DEN HARTOG J. P. Advanced Strength of Materials. New York: McGraw-Hill: 1968.

[3] TIMOSHENKO, S. P. Resistência dos Materiais, Vol II. Rio de Janeiro: LTC Editora: 1982.

[4] BORESI, A. P.; SCHMIDT R. J., SIDEBOTTOM, O. M. Advanced Mechanics of Materials. New York: John Wiley \& Sons: 1993. 